\title{
Phenotypic and genetic characterization of a family carrying two Xq21.1-21.3 interstitial deletions associated with syndromic hearing loss
}

\author{
Sandra lossa ${ }^{1,2}$, Valerio Costa ${ }^{3}$, Virginia Corvino ${ }^{4}$, Gennaro Auletta $^{4}$, Luigi Barruffo ${ }^{4}$, Stefania Cappellani ${ }^{5}$, \\ Carlo Ceglia ${ }^{2}$, Giovanni Cennamo ${ }^{6}$, Adamo Pio D'Adamo ${ }^{5,7}$, Alessandra D'Amico ${ }^{8}$, Nilde Di Paolo ${ }^{8}$, \\ Raimondo Forte ${ }^{9}$, Paolo Gasparini ${ }^{5,7}$, Carla Laria ${ }^{4}$, Barbara Lombardo ${ }^{1,2}$, Rita Malesci ${ }^{4}$, Andrea Vitale ${ }^{10}$, \\ Elio Marciano ${ }^{4}$ and Annamaria Franzè $\mathrm{e}^{2,4^{*}}$
}

\begin{abstract}
Background: Sensorineural hearing impairment is a common pathological manifestation in patients affected by X-linked intellectual disability. A few cases of interstitial deletions at Xq21 with several different phenotypic characteristics have been described, but to date, a complete molecular characterization of the deletions harboring disease-causing genes is still missing. Thus, the aim of this study is to realize a detailed clinical and molecular analysis of a family affected by syndromic X-linked hearing loss with intellectual disability.

Results: Clinical analyses revealed a very complex phenotype that included inner ear malformations, vestibular problems, choroideremia and hypotonia with a peculiar pattern of phenotypic variability. Genomic analysis revealed, for the first time, the presence of two close interstitial deletions in the Xq21.1-21.3, harboring 11 protein coding, 9 non-coding genes and 19 pseudogenes. Among these, 3 protein coding genes have already been associated with X-linked hearing loss, intellectual disability and choroideremia.

Conclusions: In this study we highlighted the presence of peculiar genotypic and phenotypic details in a family affected by syndromic $X$-linked hearing loss with intellectual disability. We identified two, previously unreported, Xq21.1-21.3 interstitial deletions. The two rearrangements, containing several genes, segregate with the clinical features, suggesting their role in the pathogenicity. However, not all the observed phenotypic features can be clearly associated with the known genes thus, further study is necessary to determine regions involved.
\end{abstract}

Keywords: Choroideremia, Hypotonia, Interstitial deletions, Intellectual disability, X-linked hearing impairment

\section{Background}

Sensorineural hearing impairment (SNHI) can be considered the most frequent form of children's hearing loss (1/1000 born) and about $60 \%$ of the cases can be ascribed to genetic causes [1]. Most of SNHI forms are non-syndromic, representing $70 \%$ of all cases, even though at least 400 known syndromes have been described. Intellectual disability (ID) is a disorder that sometimes co-occurs with sensorineural hearing impairment.

\footnotetext{
* Correspondence: franze@ceinge.unina.it

${ }^{2}$ Ceinge Biotecnologie Avanzate, Naples, Italy

${ }^{4}$ Istituto di Audiologia, Dipartimento di Neuroscienze, Scienze Riproduttive e Odontostomatologiche, Università di Napoli "Federico II", Naples, Italy Full list of author information is available at the end of the article
}

It represents one of the major handicaps affecting $1 \%$ to $3 \%$ of the general population, with onset before age 18. It is more common in males because of the high incidence of mutations in genes located on the $\mathrm{X}$ chromosome [2]. To date, more than 80 chromosome $\mathrm{X}$-linked ID genes (XLID) have been identified [3]. In most of them mutations give rise to syndromic ID forms, often accompanied by behavioral, somatic, metabolic or neurological disorders [4,5]. Syndromic forms can also result from contiguous gene deletions. In particular, deletions and rearrangements in the Xq21 have been linked to other complex syndromes including ID, deafness, choroideremia ( $\mathrm{CHM})$, seizures, and multiple congenital anomalies [6-11]. Smaller deletions lead to a milder 
clinical phenotype $[9,10,12-16]$. ID has been reported - in few cases - in association with deafness and CHM $[17,18]$. In these cases, the patients carried interstitial deletions within the Xq21. Schwartz et al. [19] mapped the deletion identified by Rosenberg and collaborators [18] at the Xq21.2-Xq21.31. More recently, a new $16 \mathrm{Mb}$ deletion in the same region has been described [20]. Nonetheless, a complete molecular characterization of such deletions at the Xq21, harboring disease-causing genes, is still missing. In this study, we describe for the first time - to the best of our knowledge - a systematic clinical, genomic and molecular analysis of a family with a complex phenotype including syndromic congenital sensorineural X-linked hearing loss. Our analysis revealed the presence of two close interstitial deletions in the Xq21.1-21.3, harboring several protein-coding genes. We also discuss the potential role of the deletions in the described phenotype.

This paper is dedicated to the memory of the Pediatrician Dr. Alfredo Pisacane (1951-2012).

\section{Case presentation}

A four-generation Italian family (Figure 1) with an Xlinked syndromic form of hearing impairment was studied. The proband (IV-1) and his brother (IV-2) had congenital bilateral hearing SNHL that had been assessed by our group a few months after birth as described in Chinetti et al. [21]. At 2 years of age both individuals showed psycho-neuro-motor disabilities too, with different degrees of severity. In the course of this study, several other clinical signs, summarized in Table 1, were observed. In detail: proband (IV-1) born in 2006, was diagnosed with bilateral severe hearing SNHL at 5 months of age. At 2 years of age, he was diagnosed with psychoneuro-motor disability. He presented also trunk and limb hypotonicity, poor motor coordination, and was unable to walk unsupported. He was fitted with hearing aids by 6 months of age, but since January 2012 has refused to use them. At present, the ID is profound with no evolutive process. Comprehension is very poor; he recognizes only the use and function of simple and familiar objects. Verbal language is absent and he use a mimic sign language with motor stereotypies. He presents a psychotic behavioral and relational disorder. He presents balance problems and flat feet too. Proband's brother (IV-2) born in 2007, was diagnosed at 5 months with a SNHL similar to the proband. Subsequently, at about 2 years of age, he was also diagnosed with trunk and limbs hypotonicity and psychoneuro-motor disability. At present, he presents a phenotype similar to that of his brother but in a much milder form. Like his brother, he recognizes only the use and function of simple and familiar objects. Verbal language and comprehension, in spite of constant hearing aid use, is poor, but better than that of his elder brother.

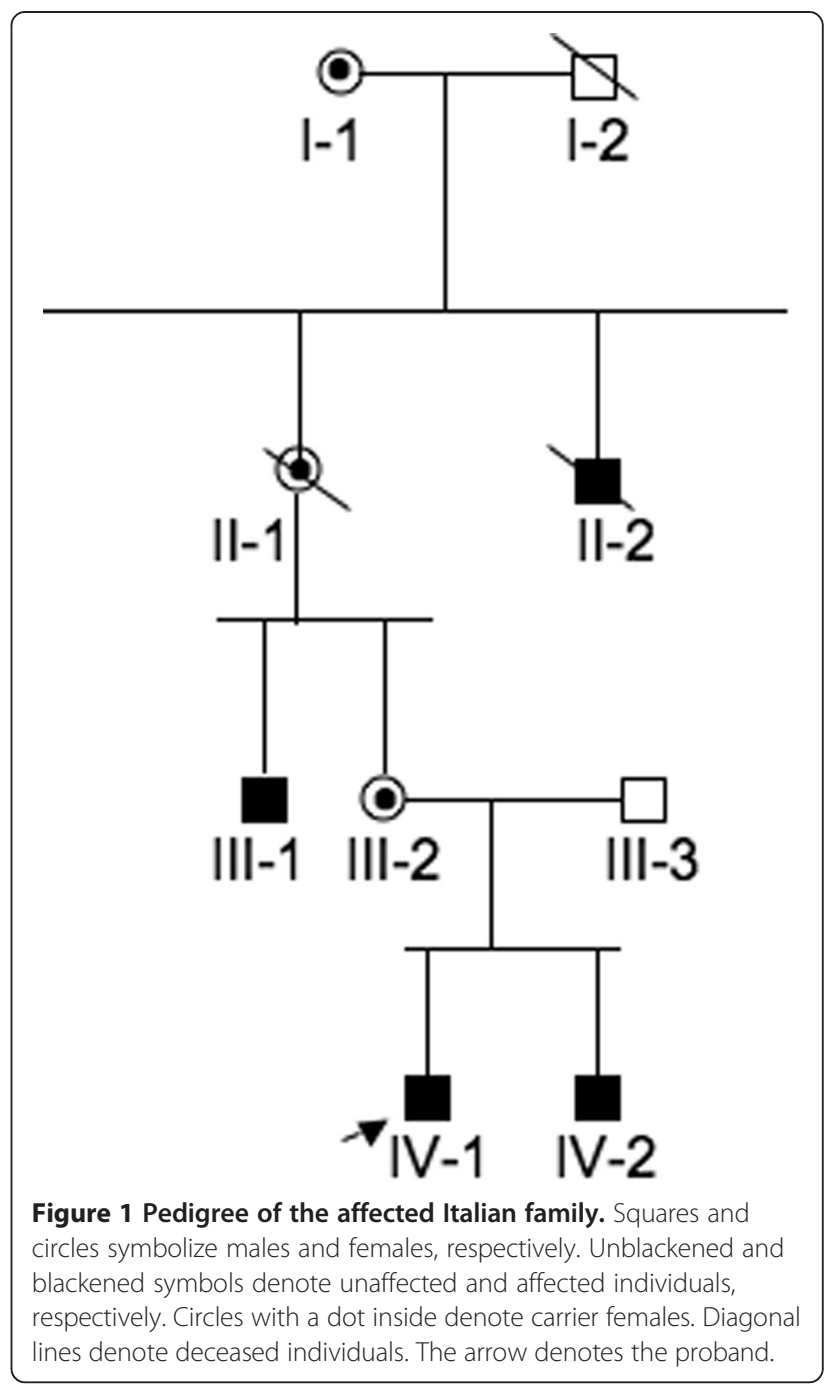

Subject III-1: Born in 1969; he was diagnosed in infancy with bilateral profound SNHL and psycho-neuromotor disability. The intellectual disability is of medium severity: he is sufficiently able to perform activities of daily living on his own. Neuropsychological tests determined a deficit that primarily occurs in short term memory for spatial material. He had been fitted with hearing aids for language development at nine years of age, and presents a simple concrete verbal language with a good verbal comprehension. Also this subject presents balance problems and flat feet but motor stereotypies are absent. Subject II-2: Born in 1947; he was diagnosed with profound bilateral hearing loss and very severe developmental delay and intellectual disability. He was not able to perform his activities of daily living unaided. Detailed clinical examinations had never been performed.

The evaluation of patients by computed tomography (CT) and magnetic resonance imaging (MRI) showed significant alterations of the inner ear apparatus, with some marked differences among affected individuals. 
Table 1 Clinical details for affected subjects

\begin{tabular}{|c|c|c|c|c|}
\hline & IV-1 & IV-2 & III-1 & II-2 \\
\hline Age (years) & 8 & 7 & 35 & - \\
\hline - SNHL & Congenital/Severe & Congenital/Severe & Congenital/Profound & $\begin{array}{l}\text { Congenital/ } \\
\text { Profound }\end{array}$ \\
\hline - Tympanometry & Normal & Normal & Normal & Normal \\
\hline - Vestibular problems & N.D. & N.D. & $\begin{array}{l}\text { Mixed sufferance: } \\
\text { central and peripheral }\end{array}$ & N.D. \\
\hline - ID & Very Severe & Severe & Moderate & Severe \\
\hline $\begin{array}{l}\text { - Age of hearing aids } \\
\text { fitting }\end{array}$ & 6 months & 6 months & 9 years & N.D. \\
\hline $\begin{array}{l}\text { - Constant use of hearing } \\
\text { aids }\end{array}$ & No & Yes & Yes & N.D. \\
\hline - Comprehension & $\begin{array}{l}\text { Very poor: recognition only of the use } \\
\text { and function of simple and familiar objects. } \\
\text { Psychotic behavioral and relational disorder }\end{array}$ & $\begin{array}{l}\text { Poor: recognition only of } \\
\text { the use and function of } \\
\text { simple and familiar objects }\end{array}$ & Good & N.D. \\
\hline - Verbal language & Absent: use of a mimic sign language & Poor & Concrete and fluent & N.D. \\
\hline $\begin{array}{l}\text { - Trunk and limbs } \\
\text { hypotonicity }\end{array}$ & Present & $\begin{array}{l}\text { Present, but milder than } \\
\text { IV:1 }\end{array}$ & Absent & N.D. \\
\hline - Motor stereotypies & Present & Present & Absent & N.D. \\
\hline - CHM evaluation & N.D. & N.D. & $\begin{array}{l}\text { Alteration typical of } \\
\text { choroideremia }\end{array}$ & N.D. \\
\hline - Brain MRI/CT & Normal & Normal & $\begin{array}{l}\text { Mega cisterna ampia. } \\
\text { Cerebral cysticercosis }\end{array}$ & N.D. \\
\hline - Ear MRI/CT & $\begin{array}{l}\text { Inner ear strongly altered. } \\
\text { Small oval window }\end{array}$ & $\begin{array}{l}\text { Inner ear strongly altered. } \\
\text { Small oval window }\end{array}$ & $\begin{array}{l}\text { Inner ear strongly altered. } \\
\text { Dysplastic fine irregularity of } \\
\text { the profile of the semicircular } \\
\text { canals }\end{array}$ & N.D. \\
\hline $\begin{array}{l}\text { - Large forehead, down } \\
\text { turned rimae palpebrarum } \\
\text { and large ears }\end{array}$ & Present & Present & Not prominent & N.D. \\
\hline - Flat feet & Present & Present & Present & N.D. \\
\hline
\end{tabular}

N.D.: not disposable.

Both CT and MRI of the siblings (IV-1, IV-2) and of the III-1 individual revealed a bulbous dilatation of the internal auditory canals (IAC), associated with the absence of the fundi of the bony plates separating the basal turn of cochlea and IAC. The modiolus was completely absent and the cochlea was dysmorphic and small, especially the medium and the apical turn. A mild dilatation of the vestibule and of the posterior semicircular canals was present in both the siblings, and proband (IV-1) also had a dilatation of the right vestibular aqueduct. In the two children, CT also revealed a small oval window that was not clearly appreciable in the individual III-1. Cochlear nerves were normal. The MRI analysis in the III-1 individual also showed a dysplastic fine irregularity in the profile of the semicircular canals, not appreciable in the other patients. Brain MRI of the subject III-1 revealed the presence of a mega cisterna magna and cerebral cysticercosis, while it did not reveal any morphological malformation in the other two patients (IV-1, IV-2) (Table 1). Figure 2 (panel 1,2) reports MRI and CT of subject IV-1. Due to the inability to communicate and poor cooperation, it was possible to perform vestibular analysis only on individual III-1. The presence of alterations found in this subject suggest both central and peripheral suffering. Indeed, for this subject, the presence of a down nystagmus was shown in all positions, which was found to be persistent, symmetric and partially inhibited by visual fixation. Vestibulo-ocular reflex (VOR) was hypoactive bilaterally and slightly reduced by visual fixation. Finally, affected subjects presented some peculiar facial characteristics too: large forehead, down turned rimae palpebrarum and large ears.

No unaffected individuals or carrier females had mental, auditory or other significant problems related to those described in the affected individuals.

\section{Results}

Clinical characteristics of the affected individuals are reported in Table 1. No abnormalities were indicated by previous high-resolution karyotype and fragile $\mathrm{X}$ molecular analyses. Thus, we first decided to perform a mutational screening in Gap junction beta-2 (GJB2), Gap junction 


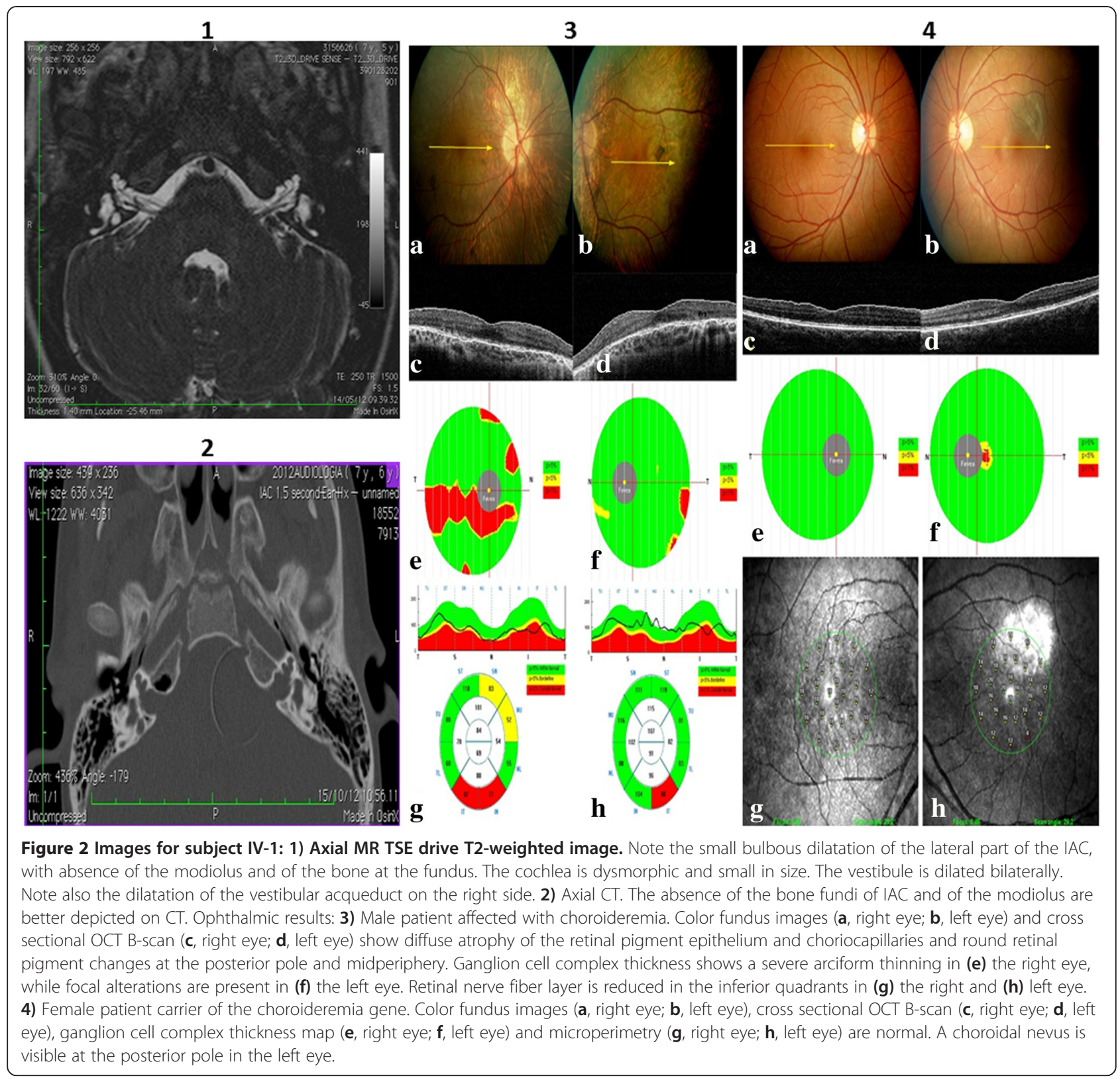

beta-6 (GJB6) and Charcot-Marie-Tooth genes: phosphoribosyl pyrophosphate synthetase 1 (PRPS1) and Gap junction beta-1 (GJB1). These analyses did not reveal the presence of any mutation. Then, we carried out a linkage analysis on this family. Unfortunately, significant results were not obtained, possibly due to the presence of a large deleted region and the consequent absence of specific markers in that region. Finally, we used a genome-wide approach, i.e. single nucleotide polymorphisms (SNPs) array analysis. Using this approach we were able to observe the presence of a deletion on $\mathrm{X}$ chromosome of about 5.2 Mb, spanning from the Xq21.1 to Xq21.31, from the nucleotide 81492309 to 86697958 (Figure 3A). Regarding protein-coding genes only, in silico analysis revealed that the deletion extends from POU class 3 homeobox 4 (POU3F4) to dachshund family transcription factor $2(D A C H 2)$ genes (Figure $3 \mathrm{C})$. Information about the coding genes mapping in the deleted region is reported in Additional file 1: Table S1_1 (see Supplementary data online S1.doc, section 1). Region-specific polymerase chain reaction (PCR) analyses on genomic DNA isolated from affected (III-1, IV-1, IV-2) and healthy carrier (III-2) individuals confirmed the absence of all these genes, and the presence of $\mathrm{SH} 3$ domain binding glutamate-rich protein like (SH3BGRL) and kelch-like family member 4 (KLHL4) genes mapping proximally to the deletion. Then, to better refine the boundaries of the deleted region we used comparative genomic 


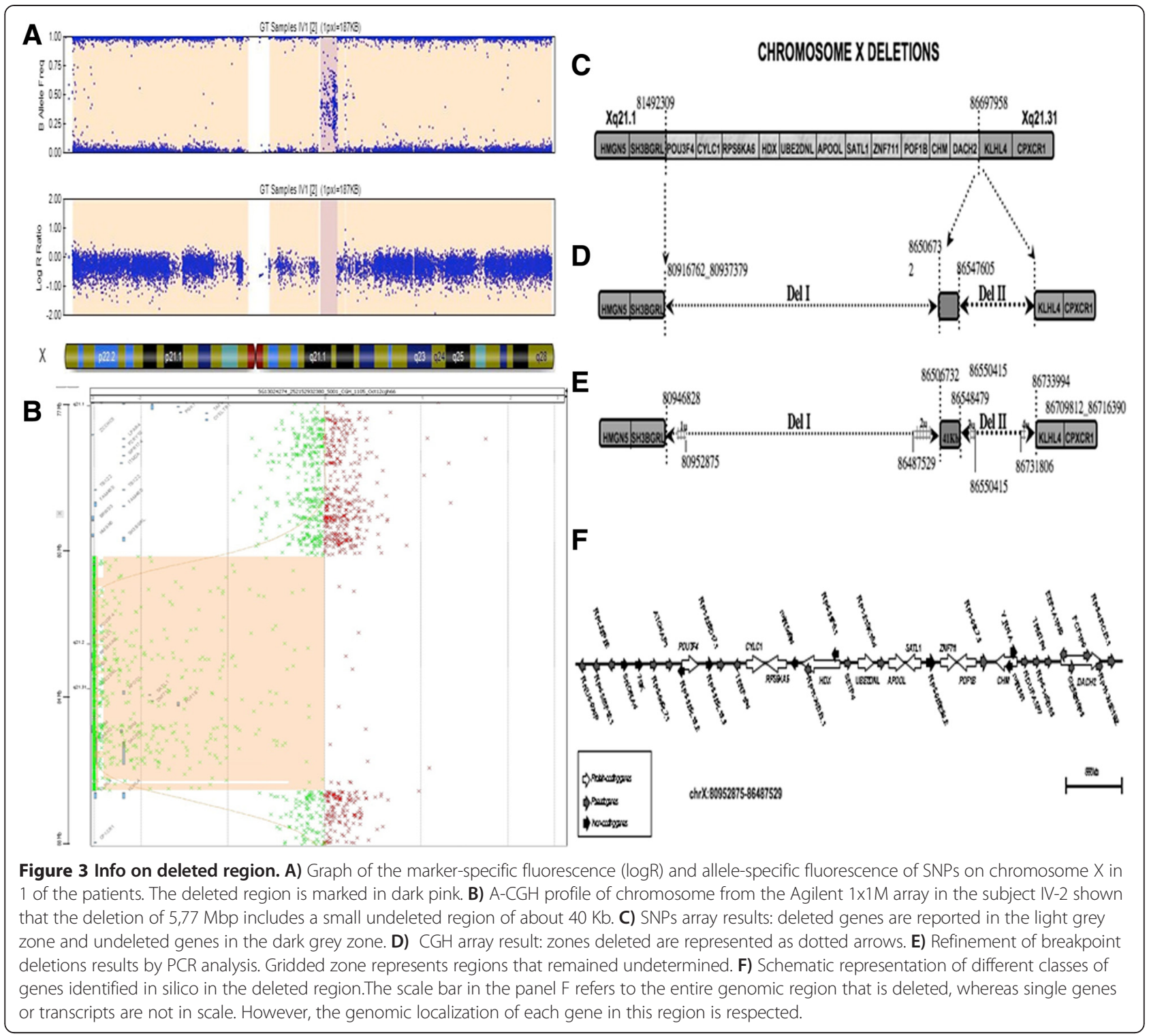

hybridization (CGH) arrays. Using this approach we confirmed the presence of a deletion of about $5.77 \mathrm{Mb},(\mathrm{chrX}$. $\left.h g 19: g .\left(80,916,762 \_80,955,540\right)\left(86,709,812 \_86,736,428\right) d e l\right)$. Interestingly, we also discovered that inside the deleted region in the proband (chrX.hg19:g.(80,916,762_80,937,379)_ $\left.\left(86,709,812 \_86,716,390\right) d e l.\right)$, a specific genomic region from the nucleotide $86,506,732$ to $86,547,605$ was not deleted (Figure 3B). This analysis indicated the presence of two close interstitial deletions, named "Del I" (of about $5.57 \mathrm{Mb}$ ) and "Del II" (of about $162 \mathrm{~Kb}$ ). These two deletions are spaced out by a region of about $41 \mathrm{~Kb}$ (Figure 3D). The presence of this region was confirmed by PCR assays (supplementary data online S1.doc, section 2: primers DelF2/DelR2 in Additional file 1: Table S1_2) carried out in all of the affected individuals (data not shown). Starting from these results, a PCR-based assay was used to further refine the two deletions' breakpoints. Given the high content of repeated regions encompassing the deleted portion of $\mathrm{X}$ chromosome, the exact sequence of some genomic regions (indicated in Figure 3E as $1 \mathrm{u}-4 \mathrm{u}$ ) is still undetermined. However, these analyses allowed us to determine that Del I consists of $5.56 \mathrm{Mb}$ (with an ambiguity of about $25 \mathrm{~Kb}$ as shown in Figure 3E, $1 \mathrm{u}+2 \mathrm{u}$ ) and Del II consists of $185.5 \mathrm{~Kb}$ (with an ambiguity of about $4 \mathrm{~Kb}, 3 \mathrm{u}+4 \mathrm{u}$ ). Browsing the main public genomic databases, we observed that the deleted region Del I contains several coding and non-coding genes (Figure 3F). In particular, the in silico analysis revealed that the patients carrying Del I completely lack the genomic region encompassing 11 protein-coding, 9 non-coding genes and 19 pseudogenes. Within this region, 3 protein-coding genes (CHM, Pou3F4 and ZNF711) have already been associated with syndromic congenital 
sensorineural X-linked hearing impairment, ID and CHM. The other deleted genes are not associated with the observed phenotypes, indicating them as potential contributors to the complex phenotype of affected individuals. No genes are annotated in the $41 \mathrm{~Kb}$ region or in Del II. By reverse transcriptase PCR (RT-PCR) on RNA isolated from peripheral blood mononuclear cells (PBMCs), we experimentally confirmed that in the III-1 individual Del I and Del II do not alter the expression of the two genes located in close proximity of the $5^{\prime}$ and $3^{\prime}$ boundaries of the identified deletions: SH3BGRL and KLHL4 (Supplementary data online Additional file 2: Figure S1).

Prior to this study, no significant visual disturbance had been noticed in any of the family subjects. Therefore, as also the CHM gene - involved in choroideremia onset was deleted, we carried out a detailed ophthalmological study on individuals III-1 and III-2. Clinical alterations typical of a CHM affected individual were observed for patient III-1 (Figure 2, panel 3), whereas a mild phenotype, typical of carrier individuals was reported in patient III-2 (Figure 2, panel 4).

\section{Discussion}

Sensorineural congenital hearing loss, vestibular problems, intellectual disability, choroideremia, hypotonia and some peculiar facial dysmorphisms have been observed in the family members (Table 1). Few studies have reported some of the phenotypic characteristics (intellectual disability, hearing loss and choroideremia) here described $[17,20]$. However, a comprehensive clinical, genomic and molecular characterization has not yet been performed. It is particularly relevant to observe the phenotypic variability in the family subjects reported in this study. In particular, the large difference in the degree of intellectual disability, which is more pronounced for proband (IV-1) compared to his brother (IV-2) and above all to their uncle (III-1). Indeed, even though individual III-1 did not undergo any treatment during his infancy he had a very milder phenotype compared to his relatives. Some differences among affected subjects have also been observed in the conformation of the inner ear and brain (Table 1).

From a molecular point of view this is the first report of two close deletions in the Xq21.1-21.3 regions: Del I and Del II (Figure 3D). This genomic region encompasses several genes, reported in detail in Figure $3 \mathrm{C}$ and Additional file 1: Table S1_1 (see Supplementary data online S1.doc, section 1). Three of them - POU3F4, ZNF711 and CHM are particularly significant for the clinical symptoms described in the affected individuals. POU3F4 has a proven role in hearing loss and inner ear malformations [22], ZNF711 has been associated with simple ID [23] and $\mathrm{CHM}$ with the choroideremia [24]. In the analyzed family, choroideremia symptoms are not particularly significant. Indeed, CHM has not been previously diagnosed in family members. CHM alterations have been determined only by instrumental ophthalmic exams (Table 1; Figure 2, panel 3 and 4). Our study also documented other peculiar phenotypic features, such as hypotonia and facial dysmorphism, in the family members. Interestingly, none of the genes present in the deletion described is clearly associated with these symptoms, as shown in Additional file 1: Table S1_1 (see Supplementary data online S1.doc, section 1).

However, although the other genes have not been associated with any of the observed pathological traits, we cannot exclude that the encoded proteins may interact with known disease-causing genes. Interestingly, browsing BioGRID - an online interaction repository - we observed that the protein encoded by RPS6KA6 gene has more than 35 interactors, with a significant involvement in "central nervous system development" and "negative regulation of mesoderm development". This observation suggests that some deleted genes may potentially encode proteins that interact, both directly and indirectly, with other proteins whose function is strictly correlated with the observed phenotypes. Other protein coding genes, not mapping to the deleted $\mathrm{X}$ chromosome region, may account for the unexpected pathological features. It indicates that further genome-wide analyses are needed to identify them. Moreover, our results highlight the presence of pseudogenes and of other non-coding genes in the deleted region. Notably, both pseudogenes and ncRNAs have been implicated in a novel regulatory networking (acting as ceRNA) and have been recently been indicated as relevant in neural cell plasticity and in neurodegenerative processes [25-28]. Therefore, we cannot exclude that the deletion of some processed pseudogenes, long ncRNAs (lncRNAs) and microRNAs (miRNAs) (Figure 3, panel F), may affect the expression of other genes, located elsewhere in the genome. Large-scale gene expression studies in these patients (by RNA-Sequencing) will clarify this crucial point. Del II deletion does not contain regulatory or coding sequences. This region may harbor a nonpathogenic copy number variation $(\mathrm{CNV})$, which may have existed before the larger deletion arose. However, browsing of public CNV databases [29] excluded the presence of rearrangements encompassing the small deletions. However, the presence of still uncharacterized regions with relevant coding or regulatory functions - in Del II cannot be excluded.

\section{Conclusions}

In this study we identified two, previously unreported, Xq21.1-21.3 interstitial deletions in a family with a syndromic form of hearing loss with variable phenotype. The two rearrangements, segregate with the clinical features in affected subjects, suggesting their role in the pathogenicity. Not all the observed phenotypic features can be clearly associated to known genes. The presence 
of several pseudogenes and non-coding genes within the deleted region may correlate to the variable phenotype observed, and potentially affect unexplored molecular pathways or involve yet underestimated epigenetic mechanisms. So, further studies are necessary to obtain a more detailed genotype-phenotype correlation.

Data herein described confirm that this region of the $\mathrm{X}$ chromosome is characterized by increased proneness to chromosome breakage.

\section{Methods}

Affected individuals IV-1, IV-2 and III-1 were analyzed by $\mathrm{CT}$ scan of ears and brain without contrast medium and by MRI of brain and ears (Philips $1.5 \mathrm{~T}$ ). Gadolinium injection was subsequently required because of a suspected associated brain cysticercosis in individual III-1. Vestibular examination was performed with videonystagmoscopy (VNS) and included testing for spontaneous and positional nystagmus (head-hanging, left and right lateral supine positions) with and without visual fixation; testing for VOR generated by passive head rotation in the horizontal plane; VOR suppression test. Ophthalmic examination included best-corrected visual acuity (BCVA) by Snellen chart, intraocular pressure tonometry, anterior segment examination and ophthalmoscopy. Combined spectral domain optical coherence tomography/microperimetry system (SD-OCT, OCT/SLO, OPKO-OTI, Miami, FL) was used to obtain fundus-related microperimetry [30,31]. For fundus-related microperimetry, we used the Polar 3 testing pattern, a stimulus size Goldmann III test spot $(\varnothing=26$ arc/min), stimulus intensity between 16 and $0 \mathrm{~dB}(10-400$ asb). The RTVue-100 SD-OCT device (Optovue, Inc., Fremont, CA, USA) was used to obtain cross sectional Bscans of the posterior pole, retinal thickness in the $3 \mathrm{~mm}$ diameter central ring of the ETDRS map (central macular thickness, CMT), thickness of the retinal ganglion cell complex (GCC) and retinal nerve fiber layer (RNFL). RTVue-100 incorporates a GCC scan mode to measure inner macular retinal layer thickness, consisting of retinal nerve fiber layer, ganglion cell layer and inner plexiform layer, centered on the fovea and covering the central macula. For the peripapillary RNFL measurements, the RNFL exam protocol was used for scan acquisition, which was measured automatically at a diameter of $3.45 \mathrm{~mm}$ around the center of the optic nerve. For mutational screening genomic DNA was extracted by conventional salt precipitation protocols from peripheral blood samples collected in EDTA-containing tubes or from buccal swabs (Gentra System, DNA Isolation Kit). Amplified PCR fragments of the exon 1 and 2 of the GJB2 gene were directly sequenced as reported elsewhere [32]. The two hearing loss-related deletions of the GJB6 gene, del (GJB6D13S1830) and del (GJB6-D13S1854), were screened using a combined detection procedure [33]. For Charcout-
Marie-Tooth genes (PRPS1, GJB1) see Supplementary data online S1.doc, section 3). SNPs analysis was performed on the genomic DNA isolated from the patient and his relatives. All samples were genotyped using the Illumina HumanCytoSNP-12 BeadChip using the standard protocol. All samples were then checked for mendelian errors using Pedcheck and Pedstat [34]. Linkage analysis was performed using an X-linked parametric model with minx and complete penetrance [35]. High resolution a-CGH analysis was performed on the genomic DNA of the patient and his relatives. DNA digestion, labeling and hybridization were performed according to the manufacturer's protocols. DNA specimens were analyzed with the Human Genome CGH Microarray kit 4x180K (Agilent Technologies, Santa Clara, CA), with an average space of $13 \mathrm{~Kb}$ and allowing an average resolution of $25 \mathrm{~Kb}$. The proband's genomic DNA was further analyzed with the Human Genome CGH Microarray kit 1xM (Agilent Technologies, Santa Clara, CA), with an average space of 2,1 $\mathrm{Kb}$. Microarrays were scanned on the Agilent G2565CA scanner and image files were quantified using Agilent's Feature Extraction software (V10.10.1.1); data were visualized with Agilent's Genomic Work Bench Standard Edition (V6.5.0.58). Primer pairs for PCR analyses were designed using the Primer3 software [36]. The primer pairs obtained were checked for non-specific hybridizations in other genome regions using the National Center for Biotechnology Information (NCBI) Basic Local Alignment Search Tool (BLAST) [37]. The different amplified fragments have a variable length due to the need to not amplify regions of the $\mathrm{X}$ chromosome frequently repeated in the entire genome, in particular, given the high presence of repeated sequences dispersed in the genomic region analyzed, we took advantage of the RepeatMask track of the USCS Genome Browser [38] to design oligonucleotide pairs to validate the deletions. Primer sequences and PCR conditions used in this study are reported in supplementary data online S1.doc, section 2). Total RNA was isolated from PBMCs. PBMCs were separated by Ficoll gradient centrifugation, placed in Trizol (Invitrogen), and stored at $-80^{\circ} \mathrm{C}$ according to the manufacturer's. RNA integrity and concentration were evaluated by using the Nanodrop (Thermo Scientific). The quality of RNA from each sample was considered good if the $260 / 280$ ratio was in between 1.8 and 2.0.

RT-PCR reaction was carried out by reverse transcription of total RNA using High Capacity cDNA Reverse Transcription Kit (Life Technologies). Primer sequences and PCR conditions on CDNA are reported in supplementary data online S1.doc, section 4). An extensive search for the presence of transcripts mapping within the genomic region encompassing the deletions has been performed retrieving the main genomic databases. Gene annotation tracks of RefSeq (release 62), Ensembl (version 74) and 
GENCODE (v19) have been loaded and visualized in the USCS Genome Browser [38]. All the transcripts, generated by alternative splicing, of protein coding and noncoding genes have been considered for the analysis. Only for genes proximal to the breakpoint, peaks of ChIP-Seq data for transcription factors (TFs) binding (GENCODE) were analyzed. The presence of binding sites for specific transcription factors was evaluated by visual inspection of ChIP-Seq tracks on Genome Browser.

The expression patterns, for both the protein and where available - the transcript, were evaluated by using The Human Protein Atlas database [39].

This research was approved by the Ethics Committee of University of Naples "Federico II" and written informed consent for participation in the study was obtained from all participants and from the parents of patients under 18 years of age before blood/saliva sampling.

\section{Additional files}

Additional file 1: Methods. PCR conditions and primers utilized for the different experiments realized in this study. Information on genes deleted.

Additional file 2: Figure S1. Semiquantitative RT-PCR assay.

\section{Competing interests}

The authors declare that they have no competing interests.

\section{Authors' contributions}

SA-Substantial contributions to acquisition, analysis and interpretation of molecular biology data and drafting of the article. VC- substantial contributions to conception and design of molecular data and critical revision of the article. GA- substantial contributions to analysis and interpretation of audiological data. LB- substantial contributions to analysis and interpretation of psychiatric data. SC- substantia contributions to acquisition of molecular data. CG- substantial contributions to acquisition and analysis of molecular data. GC-substantial contributions to analysis and interpretation of ophthalmological data. VC-substantial contributions to acquisition audiological data. APD- substantial contributions to analysis and interpretation of molecular data. AD- substantial contributions to interpretation of imaging data. NDP-substantial contributions to acquisition of imaging data. RF- substantial contributions to acquisition and analysis of ophthalmological data. PG- substantial contributions to conception and design of the study. Revision of the article critically for important intellectual content. CL- substantial contributions to acquisition of audiological data. BL-substantial contributions to analysis and interpretation of molecular data and in drafting the article. RM- substantial contributions to acquisition of audiological data. AV- substantial contributions to acquisition of molecular data. EM- substantial contributions to analysis and interpretation of audiological data. Revision of the article critically for important intellectual content. AF- substantial contributions to conception and design of the study; analysis and interpretation of data and drafting of the article. All authors read and approved the final manuscript.

\section{Acknowledgment}

The authors particularly thank the family who participated in this study. S. I. was supported by MO. DO- Model Organism grant and A.V. was supported by CREME grant.

\section{Author details}

${ }^{1}$ DMMBM, Università di Napoli "Federico II", Naples, Italy. ${ }^{2}$ Ceinge Biotecnologie Avanzate, Naples, Italy. 'IGB "A. Buzzati Traverso", CNR, Naples, Italy. ${ }^{4}$ Istituto di Audiologia, Dipartimento di Neuroscienze, Scienze Riproduttive e Odontostomatologiche, Università di Napoli "Federico II", Naples, Italy. ${ }^{5}$ Institute for Maternal and Child Health - IRCCS "Burlo Garofolo",
Trieste, Italy. "Dipartimento di Oftalmologia, Università di Napoli "Federico II", Naples, Italy. ${ }^{7}$ University of Trieste, Trieste, Italy. ${ }^{8}$ Dipartimento di Scienze Biomediche Avanzate, Università di Napoli "Federico II", Naples, Italy. ${ }^{9}$ Dipartimento di Oftalmologia Pediatrica, Università di Salerno, Salerno, Italy. ${ }^{10}$ Dipartimento di Scienze Motorie e del Benessere, Università di Napoli "Parthenope", Naples, Italy.

Received: 29 September 2014 Accepted: 17 February 2015 Published online: 20 March 2015

\section{References}

1. Rehm HL. Genetics and the Genome Project. Ear Hearing. 2003:24:270-4.

2. Leonard $H$, Wen $X$. The epidemiology of mental retardation: challenges and opportunities in the new millennium. Ment Retar Dev Disab Res Rev. 2002:8:117-34.

3. Des Portes V. X-linked mental deficiency. Handb Clin Neurol. 2013;111:297-306.

4. Kleefstra T, Hamel BCJ. X-linked mental retardation: further lumping, splitting and emerging phenotypes. Clin Genet. 2005;67:451-67.

5. Ropers HH, Hamel BC. X-linked mental retardation. Nat Rev Genet. 2005;6:46-57.

6. Ayazi S. Choroideremia, obesity, and congenital deafness. Am J Ophthalmol. 1981;92:63-9.

7. Tabor A, Andersen O, Lundsteen C, Niebuhr E, Sardemann H. Interstitial deletion in the "critical region" of the long arm of the X chromosome in a mentally retarded boy and his normal mother. Hum Genet. 1983;64:196-9.

8. Rosenberg T, Schwartz M, Niebuhr E, Yang HM, Sardemann H, Andersen O, et al. Choroideremia in interstitial deletion of the $X$ chromosome. Ophthalmic Paediatr Genet. 1986;7:205-10.

9. de Kok YJ, Vossenaar ER, Cremers CW, Dahl N, Laporte J, Hu LJ, et al. Identification of a hot spot for microdeletions in patients with $\mathrm{X}$-linked deafness type 3 (DFN3) 900 kb proximal to the DFN3 gene POU3F4. Hum Mol Genet. 1996;5:1229-35.

10. Hildebrand MS, de Silva MG, Tan TY, Rose E, Nishimura C, Tolmachova T, et al. Molecular characterization of a novel X-linked syndrome involving developmental delay and deafness. Am J Med Genet A. 2007;143A:2564-75.

11. Rush ET, Schaefer GB. Identification of an X-linked deletion syndrome through comparative genomic hybridization microarray. Semin Pediatr Neurol. 2010;17:51-3.

12. Cremers FP, van de Pol DJ, Diergaarde PJ, Wieringa B, Nussbaum RL, Schwartz M, et al. Physical fine mapping of the choroideremia locus using Xq21 deletions associated with complex syndromes. Genomics. 1989;4:41-6

13. Merry DE, Lesko JG, Sosnoski DM, Lewis RA, Lubinsky M, Trask B, et al. Choroideremia and deafness with stapes fixation: a contiguous gene deletion syndrome in Xq21. Am J Hum Genet. 1989;45:530-40.

14. Naranjo S, Voesenek K, de la Calle-Mustienes E, Robert-Moreno A, Kokotas H, Grigoriadou M, et al. Multiple enhancers located in a 1-Mb region upstream of POU3F4 promote expression during inner ear development and may be required for hearing. Hum Genet. 2010;128:411-9.

15. Anger GJ, Crocker S, McKenzie K, Brown KK, Morton CC, Harrison K, et al. X-linked deafness-2 (DFNX2) phenotype associated with a paracentric inversion upstream of POU3F4. Am J Audiol. 2014;23:1-6.

16. Choi JW, Min B, Kim A, Koo JW, Kim CS, Park WY, et al. De Novo Large Genomic Deletions Involving POU3F4 in Incomplete Partition Type III Inner Ear Anomaly in East Asian Populations and Implications for Genetic Counseling. Otol Neurotol. 2015;36:184-90.

17. Nussbaum RL, Lesko JG, Lewis RA, Ledbetter SA, Ledbetter DH. Isolation of anonymous DNA sequences from within a submicroscopic $X$ chromosomal deletion in a patient with choroideremia, deafness, and mental retardation. Proc Natl Acad Sci U S A. 1987;84:6521-5.

18. Rosenberg T, Niebuhr E, Yang HM, Parving A, Schwartz M. Choroideremia, congenital deafness and mental retardation in a family with an $X$ chromosomal deletion. Ophthalmic Paediatr Genet. 1987;8:139-43.

19. Schwartz M, Yang HM, Niebuhr E, Rosenberg T, Page DC. Regional localization of polymorphic DNA loci on the proximal long arm of the $X$ chromosome using deletions associated with choroideremia. Hum Genet. 1988;78:156-60.

20. Song MH, Lee HK, Choi JY, Kim S, Bok J, Kim UK. Clinical evaluation of DFN3 patients with deletions in the POU3F4 locus and detection of carrier female using MLPA. Clin Genet. 2010;78:524-32. 
21. Chinetti V, lossa S, Auletta G, Corvino V, De Luca M, De Falco F, et al. Mutational analysis for GJB2, GJB6, and GJB3 genes in Campania within a universal neonatal hearing screening programme. Int J Audiol. 2011;50:866-70.

22. de Kok YJ, van der Maarel SM, Bitner-Glindzicz M, Huber I, Monaco AP, Malcolm S, et al. Association between X-linked mixed deafness and mutations in the POU domain gene POU3F4. Science. 1995;267:685-8.

23. Tarpey PS, Smith R, Pleasance E, Whibley A, Edkins S, Hardy C, et al. A systematic, large-scale resequencing screen of $X$-chromosome coding exons in mental retardation. Nat Genet. 2009;41:535-43.

24. van Bokhoven $\mathrm{H}$, van den Hurk JA, Bogerd L, Philippe C, Gilgenkrantz S, de Jong $\mathrm{P}$, et al. Cloning and characterization of the human choroideremia gene. Hum Mol Genet. 1994;3:1041-6.

25. Junn E, Mouradian MM. MicroRNAs in neurodegenerative diseases and their therapeutic potential. Pharmacol Ther. 2012;133:142-50.

26. Costa V, Esposito R, Aprile M, Ciccodicola A. Non-coding RNA and pseudogenes in neurodegenerative diseases: "The (un)Usual Suspects". Front Genet. 2012:3:231.

27. Poliseno L, Salmena L, Zhang J, Carver B, Haveman WJ, Pandolfi PP. A coding-independent function of gene and pseudogene mRNAs regulates tumour biology. Nature. 2010;465:1033-8.

28. Salmena L, Poliseno L, Tay $Y$, Kats L, Pandolfi PP. A ceRNA hypothesis: the Rosetta Stone of a hidden RNA language? Cell. 2011;146:353-8.

29. Database of Genomic Variants [http://dgv.tcag.ca/dgv/app/home]

30. Landa G, Rosen RB, Garcia PM, Seiple WH. Combined three-dimensional spectral OCT/SLO topography and microperimetry: steps toward achieving functional spectral OCT/SLO. Ophthalmic Res. 2009;43:92-8.

31. Forte R, Cennamo G, Finelli ML, Bonavolontà P, Greco GM, de Crecchio G. Retinal Micropseudocysts in Diabetic Retinopathy: Prospective Functional and Anatomic Evaluation. Ophthalmic Res. 2012;2(48):6-11.

32. Chinetti V, lossa S, Auletta G, Laria C, De Luca M, Di Leva F, et al. Screening for GJB2 and GJB6 gene mutations in patients from Campania region with sensorineural hearing loss. Int J Audiol. 2010;49:326-31.

33. del Castillo FJ, Rodríguez-Ballesteros M, Alvarez A, Hutchin T, Leonardi E, de Oliveira CA, et al. A novel deletion involving the connexin-30 gene, del (GJB6-d13s1854), found in trans with mutations in the GJB2 gene (connexin-26) in subjects with DFNB1 non-syndromic hearing impairment. J Med Genet. 2005;42:588-94.

34. O'Connell JR, Weeks DE. PedCheck: A program for identifying genotype incompatibilities in linkage analysis. Am J Hum Genet. 1998;63:259-66.

35. Abecasis GR, Cherny SS, Cookson WO, Cardon LR. Merlin-rapid analysis of dense genetic maps using sparse gene flow trees. Nat Genet. 2002;30:97-101.

36. Primer3 software [http://bioinfo.ut.edu/primer3-0.4.0/]

37. BLAST [http://blast.ncbi.nlm.nih.gov/Blast.cgi]

38. Genome Browser [http://genome.ucsc.edu/]

39. Human Protein Atlas database [http://www.proteinatlas.org]

\section{Submit your next manuscript to BioMed Central and take full advantage of:}

- Convenient online submission

- Thorough peer review

- No space constraints or color figure charges

- Immediate publication on acceptance

- Inclusion in PubMed, CAS, Scopus and Google Scholar

- Research which is freely available for redistribution

Submit your manuscript at www.biomedcentral.com/submit 